\title{
COMMENT
}

\section{0 years of influenza research seen through the lens of Covid-19}

\author{
Debby Bogaert ${ }^{1}$ and David H. Dockrell ${ }^{1}$ \\ Mucosal Immunology (2020) 13:561-562; https://doi.org/10.1038/s41385-020-0291-9
}

It is perhaps a strange coincidence that in this issue of Mucosal Immunology a review entitled "Seasonal and pandemic influenza: 100 years of progress, still much to learn" is being published, while at the same time another global pandemic, this time caused by a novel coronavirus infection SARS-CoV-2, also known as Covid-19, spreads around the globe. Dunning et al. provide a comprehensive overview of current knowledge regarding seasonal influenza, with a detailed description on how the immunology of influenza has shaped the current standard of therapy and prevention. ${ }^{1}$ This review was written in the period before the world was confronted with the SARS-CoV-2 outbreak, which for many might feel a long time ago, though in reality is only 2-3 months in the past. We now live in a very different world where a novel virus has redefined how we experience essential elements of modern life ranging from work and education to travel and recreation. This has all arisen because of a rapidly spreading virus causing major morbidity and mortality, primarily due to severe pneumonia and development of acute respiratory distress syndrome (ARDS). ${ }^{2}$

Observed through the contemporary lens of covid-19, this review becomes an even more interesting and important piece of work, integrating knowledge accumulated over 100 years of research in epidemiology, global and public health, as well as evolutionary biology and immunology. These constitute the scientific rationale behind our effective current program of preventive and treatment strategies against (seasonal) influenza. It also forms a template for the knowledge, we must rapidly acquire to have similar success in controlling the novel SARS-CoV2 outbreak. The first lesson that can be distilled from this review is how difficult it still is to fully prevent seasonal influenza. With the current SARS-CoV-2 pandemic, the clock has just started to tick, and this review highlights that we need to rapidly learn about this new virus, simultaneously using our experience with previous outbreaks including influenza. The higher $R_{0}$ (reproduction number) and fatality associated with this virus however emphasize the need to learn quickly. ${ }^{3}$

The current review starts by describing the devastating effect of the influenza pandemic that raged the globe 100 years ago, with an estimated 675,000 people dying in the USA alone. ${ }^{4}$ The authors highlight how the Spanish flu pandemic caused the death of a famous artist Egon Schiele and his family. Covid-19 is already exerting a similar toll. In an era of interdisciplinary endeavor, the author, cartographer and visual artist Tim Robinson has been among the early casualties of Covid-19, dying in London a few days after the death of his wife. It is worth reflecting how ironic it is that an artist defined by his connection with empty landscapes should fall victim to a pandemic for which one of our main control measures is social distancing.

The review then addresses some of the key features of influenza pathogenesis. It provides a state-of-the-art resume of innate and adaptive immune responses but also highlights that important knowledge gaps remain. After 100 years of research important insights have helped us to understand how viruses evolve within the human and animal populations at geographical scale, under the selective pressure of immune responses and how differences in immune responses alter severity of disease. This knowledge has helped us to develop antiviral therapies and vaccination strategies. ${ }^{1}$ Yet we still face challenges identifying those patients who develop critical illness and bacterial super-infection as the authors high-light. There can also be surprises though: the author's own data shows that patients with asthma were less likely to present with severe pulmonary or systemic disease when exposed to H1N1pdm09.'

When faced with a brand new virus like SARS-CoV-2 rapidly emerging in the human population, we have a much more limited knowledge base to work with, leaving us at a significant disadvantage. We rapidly must understand the origin, genetic variation and epidemiological characteristics of the new virus. This includes knowledge on mode of transmission, incubation period, window of transmission, and reproductive number, to ensure an effective public health response and to put in place appropriate infection control measures. As a consequence, most nations, supported by organizations like WHO and GOARN, have emergency response plans in place that can deal with emerging major outbreaks, including pandemic influenza and other emerging respiratory viruses (https://extranet.who.int/goarn/).

In contrast to the array of data succinctly summarized by the authors on influenza, we lack comparable understanding for Covid-19. However, what we already have learned is the importance of the human angiotensin-converting enzyme 2 (hACE2) as the entry receptor for SARS-CoV- $2 .^{5}$ Based on genetic information researchers have identified that the biochemical and structural properties of SARS-CoV-2 receptor binding domain (RBD) show an even higher hACE2-binding affinity compared to SARS-CoV, ${ }^{6}$ which might help to explain the higher transmission rates and infectivity. The paucity of post-mortem data and the infectivity of invasive procedures such as bronchoscopies are two barriers to acquiring information, but despite many hurdles, information is rapidly accumulating. We still know little about immunity in mild disease, including correlates of effective immunity such as neutralizing antibody, though preliminary data show that antibody responses kick in early in the disease process,

${ }^{1}$ Center for Inflammation Research, University of Edinburgh, Edinburgh, UK

Correspondence: Debby Bogaert (d.bogaert@ed.ac.uk)

Received: 16 April 2020 Accepted: 16 April 2020

Published online: 1 May 2020 
with $\lg A$ and $\lg M$ detectable within 5 days after start of symptoms. ${ }^{7}$ In addition, first evidence has been obtained suggesting antibodies from recovered cases are able to neutralize viral entry, and protect against re-infection. ${ }^{5}$ Interestingly, in more severe cases lymphopenia is profound and associated with severity and mortality, which is likely a consequence of activation of apoptosis. One early transcriptomic analysis has suggested activation of $\mathrm{p} 53$ signaling pathways, but other pathways are likely to be involved as well. ${ }^{8}$ In the subset of patients with severe disease accompanied by respiratory failure or acute respiratory distress syndrome, a small numbers of post-mortem studies have also shown increased numbers of Th17 cells and activated CD8+ T-cells with expression of granzymes and perforin. ${ }^{9}$ There is also evidence of prominent macrophage activation in a subset of patients, with a high level of ferritin and IL-6. In some, this may be extreme, resembling macrophage activation syndrome, which frequently has a viral trigger, but this time associated with a merely mucosal rather than systemic immune activation. ${ }^{10}$ We recognize many of those with severe disease have medical comorbidity, in particular hypertension or advanced age, but we need to understand how these conditions influence susceptibility and we need to analyze genetic studies to help explain the reason why otherwise healthy individuals can have a severe outcome including death.

Some of the patterns of dysregulated inflammation and alterations in immune cell populations are similar to those in sepsis. This raises the possibility that there is a role for co-infection or microbiota-driven inflammation in severity of disease. Coinfections contribute significantly to morbidity and mortality in influenza. ${ }^{1}$ Early data from China suggested co-infection in as many as $50 \%$ of deceased SARS-CoV-2 patients, ${ }^{10}$ while other reports in milder disease suggest co-infection in $10 \%$ or less. Details on pathogens associated with co-infection are limited, but Chen et al. described complex infections with a combination of Gram-negative pathogens identified, as well as fungi in a few cases. $^{2}$ In addition to consequences for antimicrobial selection when required, learning about these patterns will be important in promoting preventive strategies such as vaccination. Bacterial vaccines, specifically those directed against the pneumococcus, are a cornerstone of prevention of influenza-related morbidity and mortality especially in vulnerable groups, at times of seasonal or pandemic influenza. ${ }^{1}$ Research is needed to establish if management of (mixed) bacterial infections over the course of disease is also important for successful treatment of Covid-19, so we can consider these alternative preventive strategies.

Management of seasonal influenza has benefitted greatly from the development of antiviral therapies as the authors summarize in their review. ${ }^{1}$ For Covid-19, initial strategies are exploring the efficacy of antivirals, which have been developed in other settings and repurposed anti-inflammatory or immune modulating drugs. In parallel, many groups throughout the world have embarked on the challenging task of rapidly developing a SARS-CoV-2 vaccine, a process that is dramatically accelerated by the use of novel technologies designed to accelerate development of vaccines. ${ }^{11}$ Challenges will remain though, regarding how immunogenic the epitopes derived from the spike proteins of SARS-COV-2 are, how well how epitopespecific antibodies neutralize virus, how long-lasting acquired immunity is and whether there is any risk of antibodydependent enhancement of disease, as has been demonstrated for SARS and other coronaviruses. ${ }^{12}$ It will take time to ensure safety and efficacy, as well as to scale up vaccine production for a global demand. A coordinated multisector effort has been put into place at a pace and depth never observed before. But it remains to be seen whether this virus becomes endemic, and whether SARS-CoV-2 will be the last coronavirus that jumps from undefined intermediate hosts to man.

History suggests that the battle against SARS-CoV-2 and related coronaviruses is still in its infancy. Once this new Coronavirus outbreak has been overcome, we may come to discover we have fought only one battle not the entire war. We must learn lessons not only about preparedness, but also about specifics of immunity to this virus, and more generally about mechanisms underpinning severe lung infection, including the role of co-infection and immune dysregulation. This will help to effectively combat the ongoing threat of pandemic respiratory viruses. We need to be better prepared next time, and whatever it takes, and how much time passes before the next pandemic arrives, we must not let our guard down. We must continue to learn from past and future influenza outbreaks, as well as novel respiratory virus pandemics such as covid-19. Each of these teach us important lessons. This will help us prepare more effectively for the next pandemic respiratory virus. It is certain they will come, even though we cannot predict when and where they will emerge.

\section{ADDITIONAL INFORMATION}

Competing interests: The authors declare no competing interests.

Publisher's note Springer Nature remains neutral with regard to jurisdictional claims in published maps and institutional affiliations.

\section{REFERENCES}

1. Dunning, J., Thwaites, R., Openshaw, P. Seasonal and pandemic influenza: 100 years of progress, still much to learn. Mucosal. Immunol. 2020. https://doi.org/ 10.1038/s41385-020-0287-5. [Epub ahead of print].

2. Chen, N. et al. Epidemiological and clinical characteristics of 99 cases of 2019 novel coronavirus pneumonia in Wuhan, China: a descriptive study. Lancet 395, 507-513 (2020).

3. Wang, Y., Wang, Y., Chen, Y., \& Qin, Q. Unique epidemiological and clinical features of the emerging 2019 novel coronavirus pneumonia (COVID-19) implicate special control measures. J. Med. Virol. 2020. https://doi.org/10.1002/jmv.25748.

4. Morens, D. M., Taubenberger, J. K. \& Fauci, A. S. Predominant role of bacterial pneumonia as a cause of death in pandemic influenza: implications for pandemic influenza preparedness. J. Infect. Dis. 198, 962-970 (2008).

5. Hoffmann, M., et al, SARS-CoV-2 cell entry depends on ACE2 and TMPRSS2 and is blocked by a clinically proven protease inhibitor. Cell. 2020;181:271-280.e8. https://doi.org/10.1016/j.cell.2020.02.052. E-pub ahead of print

6. Shang, J., et al Structural basis of receptor recognition by SARS-CoV-2. Nature. 2020. https://doi.org/10.1038/s41586-020-2179-y.

7. Guo, L., et al. Profiling early humoral response to diagnose novel coronavirus disease (COVID-19). Clin. Infect. Dis. 2020. https://doi.org/10.1093/cid/ciaa310.

8. Xiong, Y. et al. Transcriptomic characteristics of bronchoalveolar lavage fluid and peripheral blood mononuclear cells in COVID-19 patients. Emerg. Microbes Infect. 9, 761-770 (2020).

9. Xu, Z. et al. Pathological findings of COVID-19 associated with acute respiratory distress syndrome. Lancet Respir. Med 8, 420-422 (2020).

10. Huang, C. et al. Clinical features of patients infected with 2019 novel coronavirus in Wuhan, China. Lancet 395, 497-506 (2020).

11. Lu, S. Timely development of vaccines against SARS-CoV-2. Emerg. Microbes Infect. 9, 542-544 (2020)

12. Jaume, M. et al. Anti-severe acute respiratory syndrome coronavirus spike antibodies trigger infection of human immune cells via a $\mathrm{pH}$ - and cysteine proteaseindependent FcyR pathway. J. Virol. 85, 10582-10597 (2011). 